\title{
Galectin-3 Targeting in Thyroid Orthotopic Tumors Opens New Ways to Characterize Thyroid Cancer
}

\author{
Francesco De Rose ${ }^{1}$, Miriam Braeuer ${ }^{1}$, Sten Braesch-Andersen ${ }^{2}$, Angela M. Otto ${ }^{3}$, Katja Steiger ${ }^{4,5}$, Sybille Reder ${ }^{1}$, \\ Sabine Mall ${ }^{6}$, Stephan Nekolla ${ }^{1}$, Markus Schwaiger ${ }^{1}$, Wolfgang A. Weber ${ }^{1}$, Armando Bartolazzi ${ }^{7,8}$, \\ and Calogero D'Alessandria ${ }^{1}$
}

${ }^{I}$ Nuklearmedizinische Klinik und Poliklinik, Klinikum rechts der Isar, Technische Universität München, München, Germany; ${ }^{2}$ Mabtech AB Research Laboratory, Stockholm, Sweden; ${ }^{3}$ Munich School of Biomedical Engineering, Technische Universität München, Garching, Germany; ${ }^{4}$ Institute of Pathology, Klinikum rechts der Isar, Technische Universität München, München, Germany; ${ }^{5}$ Comparative Experimental Pathology, Klinikum rechts der Isar, Technische Universität München, München, Germany; ${ }^{6}$ III, Medical Department, Klinikum rechts der Isar, Technische Universität München, München, Germany; ${ }^{7}$ Pathology Research Laboratory, Cancer Center Karolinska, Karolinska Hospital, Stockholm, Sweden; and ${ }^{8}$ Pathology Research Laboratory, Sant'Andrea University Hospital, Rome, Italy

Preoperative characterization of thyroid nodules is challenging since thyroid scintigraphy fails to distinguish between benign and malignant lesions. Galectin-3 (gal-3) is expressed in well-differentiated and in undifferentiated thyroid cancer types but not in normal thyrocytes and benign thyroid lesions. Herein, we aimed to validate gal-3 targeting as a specific method to detect non-radioiodine-avid thyroid cancer in thyroid orthotopic tumor models. Methods: Papillary (BcPAP) and anaplastic (CAL62 and FRO82-1) thyroid carcinoma cell lines were characterized via Western blot and polymerase chain reaction for gal-3 and sodium-iodide symporter (NIS) expression. An ${ }^{89} \mathrm{Zr}$-labeled $\mathrm{F}\left(\mathrm{ab}^{\prime}\right)_{2}$ antigal-3 was generated and characterized for binding versus ${ }^{125}$ I on 2 - and 3 -dimensional cell cultures. The thyroid carcinoma cells were inoculated into the left thyroid lobe of athymic nude mice, and the orthotopic tumor growth was monitored via ultrasound and fluorescence molecular tomography. Head-to-head PET/CT comparison of ${ }^{124}$ I versus ${ }^{89} \mathrm{Zr}$-deferoxamine (DFO)-F( $\left(\mathrm{ab}^{\prime}\right)_{2}$ antigal-3 was performed, followed by biodistribution studies and immunohistochemical analysis for gal-3 and NIS expression. Results: The thyroid carcinoma cells investigated were invariably gal-3-positive while presenting low or lost NIS expression. ${ }^{89} \mathrm{Zr}$-DFO-F $\left(\mathrm{ab}^{\prime}\right)_{2}$ antigal-3 tracer showed high affinity to gal3 (dissociation constant, $\sim 3.9 \mathrm{nM}$ ) and retained immunoreactivity $(>75 \%)$ on 2-dimensional cell cultures and on tumor spheroids. 125I internalization in FRO82-1, BcPAP, and CAL62 was directly dependent on NIS expression, both in 2-dimensional and tumor spheroids. PET/CT imaging showed ${ }^{89} \mathrm{Zr}-\mathrm{DFO}-\mathrm{F}\left(\mathrm{ab}^{\prime}\right)_{2}$ antigal-3 signal associated with the orthotopically implanted tumors only; no signal was detected in the tumor-free thyroid lobe. Conversely, PET imaging using ${ }^{124}$ I showed background accumulation in tumor-infiltrated lobe, a condition simulating the presence of non-radioiodine-avid thyroid cancer nodules, and high accumulation in normal thyroid lobe. Imaging data were confirmed by tracer biodistribution studies and immunohistochemistry. Conclusion: A specific and selective visualization of thyroid tumor by targeting gal-3 was demonstrated in the absence of radioiodine uptake. Translation of this method into the clinical setting promises to improve the management of patients

Received Aug. 9, 2018; revision accepted Oct. 10, 2018.

For correspondence or reprints contact: Calogero D'Alessandria, Nuklearmedizinische Klinik und Poliklinik, Klinikum rechts der Isar, Technische Universität München, Ismaninger Strasse 22, 81675 München, Germany.

E-mail: calogero.dalessandria@tum.de

Published online Oct. 25, 2018.

COPYRIGHT (C 2019 by the Society of Nuclear Medicine and Molecular Imaging. by avoiding the use of unspecific imaging methodologies and reducing unnecessary thyroid surgery.

Key Words: galectin-3; thyroid tumor; PET imaging; ${ }^{124}$; orthotopic models

J Nucl Med 2019; 60:770-776

DOI: 10.2967/jnumed.118.219105

$\mathbf{T}$ hyroid cancer is the most common endocrine cancer in the United States. Although the age-adjusted incidence of many cancers has significantly decreased during the last $10 \mathrm{y}$, a significant increase in thyroid cancer has been reported, with a reported growing incidence in men and women since 2005 tripling from 1983 to 2012 (1,2). Because the mortality of thyroid cancer has not changed during the same period, and there were no fundamental changes in thyroid cancer therapy, it is generally believed that this marked rise in incidence is caused by an increasing detection of small and clinically insignificant thyroid nodules by ultrasound imaging. To address this "thyroid cancer epidemic," the criteria for biopsy of thyroid nodules have been revised, and biopsies of small, incidentally detected nodules with morphologically benign features are now discouraged. Furthermore, some thyroid neoplasms (encapsulated follicular neoplasms with papillary features) that were previously classified as malignant are now considered benign (3). These changes have recently led to a slowing of the thyroid cancer incidence. However, the clinical criteria for performing biopsies are far from perfect and are based mostly on the size of the nodule. Thus, they may also lead to delayed diagnosis of more aggressive thyroid malignancies. Although new classifications of thyroid neoplasms on histopathology avoid overtreatment (such as total thyroidectomy, lymph node dissection, and radioiodine therapy), it would be preferable if clinically insignificant tumors could be diagnosed before surgery.

Differentiation between benign and malignant thyroid nodules is a particular problem in countries with iodine deficiency and a high prevalence of nodular goiter. A combination of ultrasound and thyroid scintigraphy with ${ }^{99 \mathrm{~m}} \mathrm{Tc}$-pertechnetate or ${ }^{123} \mathrm{I}$ is routinely used to characterize thyroid nodules, but their accuracy is limited to multinodular goiter due to reduction or loss of sodium-iodide 
symporter (NIS) expression in thyroid cancer cells, as a result of oncogenic activation (4). Therefore, there is an unmet clinical need for more accurate diagnostic tests to differentiate between benign and malignant thyroid nodules and to characterize thyroid cancer.

Galectin-3 (gal-3) is a well-established histologic marker of thyroid cancer that is not expressed by normal thyroid cells. We have previously shown that radiolabeled antibodies directed against gal-3 accumulate in subcutaneous thyroid cancer xenografts in mice and allow for high-contrast PET imaging $(5,6)$.

The purpose of this study was to further demonstrate the specificity of thyroid cancer detection by gal-3-targeting antibodies in orthotopic tumor models, characterized by low or lost NIS expression, using PET and fluorescence imaging. We showed that our methodology is highly sensitive in distinguishing specifically between normal thyroid tissue and thyroid cancer tissue, opening new possibilities for a personalized therapeutic approach to patients affected by thyroid cancer.

\section{MATERIALS AND METHODS}

\section{Western Blotting}

Cell lysates derived from 2-dimensional (2D) and 3-dimensional (3D) cultures of BcPAP (papillary), FRO82-1, and CAL62 (anaplastic) were prepared using radioimmunoprecipitation assay buffer and analyzed via Western blot as previously reported (6). The following primary antibodies were used: a rat monoclonal antibody (mAb), antigal-3 (1:5,000, M3/38; Mabtech); a mouse mAb, anti-NIS (1:500, AB17052; Abcam); a rabbit mAb, anti-thyroid transcription factor 1 (1:500, 07-60; Millipore); and a mouse mAb, anti-glyceraldehyde-3phosphate dehydrogenase (GADPH) (1:6,000, CB1001; Calbiochem). Gal-3 and thyroid transcription factor 1 were detected via a colorimetric method using a goat antirat polyclonal antibody (1:5,000, A8438; Sigma) and a goat antirabbit mAb (1:5,000, AP307A; Millipore) alkaline phosphatase-conjugated secondary antibodies, incubating the blotting membrane with 5-bromo-4-chloro-3-indolyl-phosphate/nitro blue tetrazolium chromogen (Abcam) for $5 \mathrm{~min}$. The signal corresponding to the human NIS expression was detected using a goat $\mathrm{mAb}$ antimouse horseradish peroxidase-conjugated (1:5,000, AP124P; Millipore) and chemoluminescence (enhanced chemiluminescence kit, Amersham Corp.) by exposing the blotting membrane for $20 \mathrm{~min}$ to a Hyperfilmenhanced chemiluminescence (Amersham Corp.). The Amersham enhanced chemiluminescence full-range rainbow marker (GE Healthcare) and GADPH were used as references.

\section{Real-Time Quantitative Polymerase Chain Reaction (PCR)}

To investigate gal-3 and NIS gene expression via PCR analysis, the complementary DNA was synthetized from total RNA of tumor cell and human thyrocytes (Takara Bio Europe). Primers used for the amplification of specific sequences are listed in Supplemental Table 1 (supplemental materials are available at http://jnm.snmjournals.org). After the amplification, a $10-\mu \mathrm{L}$ volume of each PCR product was separated via electrophoresis on a $1 \%$ agarose gel and stained with ethidium bromide. The bands were visualized using an Omega Lum C Imaging System (Gel Co.). A densitometric analysis of the signals was performed using the ImageJ software, and results were normalized to GADPH gene.

Quantitative PCR was performed using a StepOne Real-Time PCR System (Applied Biosystem, Life Technologies) with PowerUp SYBR Green Master Mix (BioRad) and the primers mentioned above. Data were analyzed using the $2^{-\Delta \Delta C_{\mathrm{T}}}$ method normalizing for the GADPH $C_{\mathrm{T}}$ value (7).

\section{Probes for Gal-3 Targeting}

A $\mathrm{F}\left(\mathrm{ab}^{\prime}\right)_{2}$ to gal-3 was prepared by pepsin digestion of a full rat $\mathrm{mAb}$ to gal-3 (clone M3/38; Mabtech) preconditioned as previously reported $(8,9)$.
To perform fluorescence microscopy and fluorescence molecular tomographic (FMT) imaging, the $\mathrm{F}\left(\mathrm{ab}^{\prime}\right)_{2}$ to gal-3 was conjugated to AlexaFluor 488-tetrafluorophenyl dye (GE Healthcare) and to Cy5.5$N$-hydroxysuccinimide (GE Healthcare) and the ratios dye/F( $\left.\mathrm{ab}^{\prime}\right)_{2}$ to gal-3 were measured via an Implen nanophotometer (Implen) analyzing samples at specific wavelengths (supplemental data).

For gal-3 immuno-PET targeting, the $\mathrm{F}\left(\mathrm{ab}^{\prime}\right)_{2}$ was functionalized with the chelator desferrioxamine-isothiocyanate (DFO-NCS) and labeled with ${ }^{89} \mathrm{Zr}$, followed by integrity and stability studies performed as previously described $(10,11)$.

\section{In Vitro lodine Uptake}

Monolayer cell cultures were incubated with $74 \mathrm{kBq}$ of carrier-free ${ }^{125} \mathrm{I}-\mathrm{NaI}$ as reported elsewhere (12). BcPAP, CAL62, and FRO82-1 spheroids, produced seeding $150,000,100,000$, and 50,000 cells per well, were incubated for $2 \mathrm{~h}$ with $74 \mathrm{kBq}$ of carrier-free ${ }^{125} \mathrm{I}-\mathrm{NaI}$. Control cell cultures were incubated with $300 \mu \mathrm{M} \mathrm{KClO}_{4}$ solution to measure the unspecific iodine uptake. After incubation, spheroids were centrifuged and washed, and the pellet-bound activity was counted in a $\gamma$-counter.

\section{In Vitro Cell-Binding Test of ${ }^{89} \mathrm{Zr}$-Deferoxamine (DFO)-aGal3-F(ab') 2}

Binding affinity of ${ }^{89} \mathrm{Zr}-\mathrm{DFO}-\mathrm{aGal} 3-\mathrm{F}\left(\mathrm{ab}^{\prime}\right)_{2}$ and dissociation constant and maximum binding values were determined on $2 \mathrm{D}$ cell cultures as previously described (6). The immunoreactivity of ${ }^{89} \mathrm{Zr}$ DFO-aGal3-F( $\left(\mathrm{ab}^{\prime}\right)_{2}$ was assessed by the method of Lindmo et al. (13).

The cellular internalization of ${ }^{89} \mathrm{Zr}-\mathrm{DFO}-\mathrm{aGal} 3-\mathrm{F}\left(\mathrm{ab}^{\prime}\right)_{2}$ was studied on $2 \mathrm{D}$ cell cultures and by measuring the internalized activity at different time points. Binding to tumor spheroids was tested by incubating spheroids of different cell numbers with ${ }^{89} \mathrm{Zr}$-DFO-aGal3$\mathrm{F}\left(\mathrm{ab}^{\prime}\right)_{2}$. Cell-associated activity was counted in a $\gamma$-counter.

The binding of aGal3-F( $\left.\mathrm{ab}^{\prime}\right)_{2}$ to tumor spheroids was characterized by incubation for $24 \mathrm{~h}$ at $37^{\circ} \mathrm{C}$ with AlexaFluor 488-conjugated $\mathrm{F}\left(\mathrm{ab}^{\prime}\right)_{2}$ and fluorescent images acquired at different time points using a Biorevo 9000E (Keyence) digital microscope.

\section{Orthotopic Thyroid Cancer Establishment and Monitoring}

Six-week-old pathogen-free female athymic nude-Foxn $1^{n u / n u}$ mice (Charles River Laboratories) were transplanted with thyroid cancer cells as described elsewhere (14), with slight modifications. Establishment of the orthotopic xenograft models and in vivo experimental protocols were approved by the local authorities (Regierung von Oberbayern, Germany; license 55.2-1-54-2532-216-15).

The tumor growth was monitored weekly via ultrasound scanning using a Vevo2100 Imaging System (Visualsonics) equipped with a MS550D transducer (40-MHz center frequency, focal depth of $4 \mathrm{~mm}$ ).

The presence of a tumor was confirmed by FMT imaging performed $48 \mathrm{~h}$ after injection of about $54 \mu \mathrm{g}$ of Cy5.5-labeled aGal3-F(ab' $)_{2}$ ( 2 nmol of near-infrared dye), using an FMT2500 system (VisEn Medical Inc.). Image reconstruction and analysis were performed by VisEn FMT 2500 software.

\section{I PET/CT Versus Immuno-PET Imaging of Orthotopic Tumors}

When the tumors reached $3-5 \mathrm{~mm}$ in diameter, 3 groups of mice ( 3 mice per group) bearing the different tumors were injected via a catheter in the tail vein with $1.10 \pm 0.01 \mathrm{MBq}$ of ${ }^{124} \mathrm{I}$ (IBA, PerkinElmer) in $300 \mu \mathrm{L}$ of $0.9 \% \mathrm{NaCl}$. One group of 3 healthy mice injected with the same activity was used as a control. One hour after injection, the animal, anesthetized with $5 \% \mathrm{v} / \mathrm{v}$ isoflurane $/ \mathrm{O}_{2}$, underwent a 30-min PET/CT static acquisition using an Inveon small-animal PET/CT scanner (Siemens). Characterization of malignant (tumor-bearing) versus normal (tumor-free) thyroid 


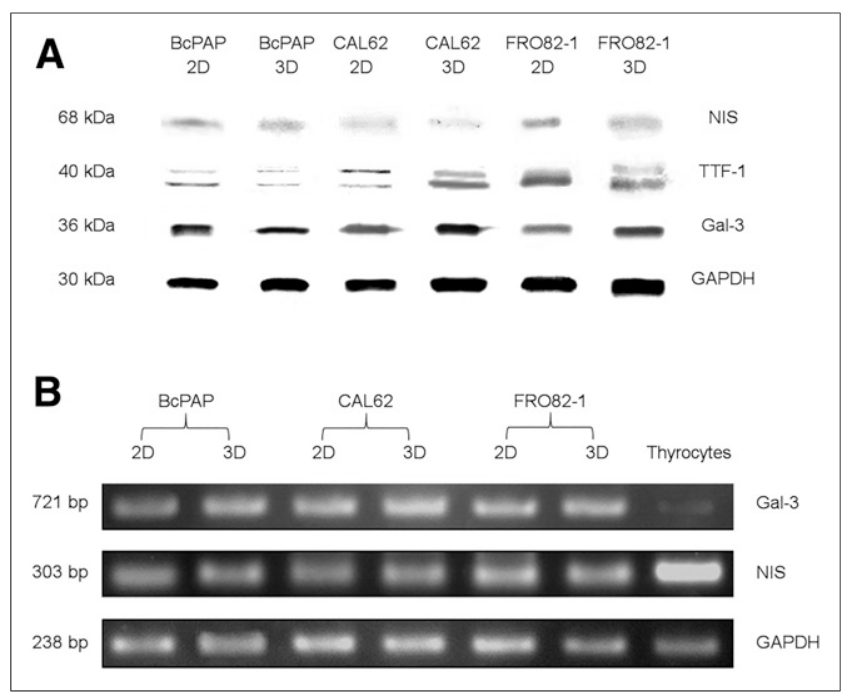

FIGURE 1. $2 \mathrm{D}$ and $3 \mathrm{D}$ cell culture characterization. (A) Cell lysates from monolayer (2D) and spheroid (3D) cell cultures analyzed via Western blot. Bands were separated with $100 \mathrm{~V}$ for $90 \mathrm{~min}$. (B) Electrophoretic separation of complementary DNA encoding for gal-3 and human NIS on $1 \%$ agarose gel, using $80 \mathrm{~V}$ for $60 \mathrm{~min}$, and staining with ethidium bromide. TTF $=$ thyroid transcription factor.

was performed by immuno-PET targeting of gal-3. Two groups of mice per orthotopic tumor type were studied ( 5 mice per tumor type), and 2 groups of healthy mice ( 5 mice per group) were used as control.

All mice were injected via a catheter in the tail vein with $2.2 \pm 0.2$ $\mathrm{MBq}$ of ${ }^{89} \mathrm{Zr}-\mathrm{DFO}-\mathrm{aGal} 3-\mathrm{F}\left(\mathrm{ab}^{\prime}\right)_{2}$ in $250 \mu \mathrm{L}$ of sodium acetate buffer
( $\mathrm{pH} 5.5$ ), and $48 \mathrm{~h}$ afterward, the mice were anesthetized and imaged via a 30-min PET/CT static acquisition. Images were reconstructed using an ordered-subsets expectation-maximization 3D maximum a posteriori algorithm. Data were normalized and corrected for randoms, dead time, and decay with no correction for attenuation or scatter.

\section{Tracer Accumulation Studies}

After each imaging session, the tracer accumulation in selected organs was measured ex vivo as previously described (6) and expressed as percentage injected dose per gram of tissue $(\% \mathrm{ID} / \mathrm{g})$.

In vivo tumor uptake of ${ }^{89} \mathrm{Zr}-\mathrm{DFO}-\mathrm{aGal} 3-\mathrm{F}\left(\mathrm{ab}^{\prime}\right)_{2}$ was analyzed using Inveon Research Workplace software (Siemens). An approximate region of interest was drawn on the left thyroid lobe encompassing the tumor signal using a threshold of $50 \%$. The $\% \mathrm{ID} / \mathrm{g}$ was calculated as ratio of mean radioactivity in each region of interest (MBq) per gram of tumor (weighed postmortem) and injected radioactivity (MBq). In vivo and ex vivo accumulation data were compared for correlation analysis. The right thyroid served as an internal control.

A dosimetry study was performed on 1 group of mice, analyzing the data of the 30-min static acquisition at $12,24,48$, and $70 \mathrm{~h}$ using OLINDA/EXM as described elsewhere (15).

\section{Histology and Immunohistochemistry}

Excised tumors were fixed in $10 \%$ neutral-buffered formalin solution for $48 \mathrm{~h}$, dehydrated under standard conditions (Leica ASP300S), and embedded in paraffin. Serial 3- $\mu \mathrm{m}$-thick sections were prepared using a rotary microtome (HM355S; ThermoFisher Scientific). Tissue slides were deparaffinized and pretreated with citrate buffer, $\mathrm{pH} 6$, for 20 or $30 \mathrm{~min}$, for gal-3 and NIS immunostaining. Immunohistochemistry applying a primary horseradish peroxidase-conjugated rat mAb antigal-3 (Mabtech) or rabbit polyclonal antibody anti-NIS (GTX37599; Genetex) was performed using a BondMax RXm system (Leica). For NIS staining, secondary species-specific polyclonal antisera with a polymer refine detection kit was applied. Immunoreactivity was visualized with 3,3'-diaminobenzidine for both antibodies. Tumor sections were counterstained with hematoxylin-eosin, and normal thyroid tissue served as a control. All hematoxylin-eosin and immunohistochemical slides were evaluated by a certified pathologist masked to the animal groups. All slides were scanned with a Leica AT2 system to a Leica eSlide Manager database and evaluated with Leica ImageScope software.

\section{Statistical Analyses}

Differences in tumor radioactivity uptake between different groups of mice were statistically analyzed with GraphPad Prism 4.0 software using the Student $t$ test for unpaired data. Two-sided significance levels were calculated and $P$ values of less than 0.05 or less than 0.01 were considered statistically significant.

\section{RESULTS}

\section{Gal-3 and Human NIS Expression}

Monolayer and 3-dimensional cultures of BcPAP, FRO82-1, and CAL62 cells showed gal-3 expression, with a band at 31 $\mathrm{kDa}$ (Fig. 1A). The relative signal density of gal-3 was about $38 \%$ higher in the spheroids than in monolayer cultures (Supplemental Fig. 1A). Human NIS expression analysis revealed a band at $68 \mathrm{kDa}$, which was weaker 


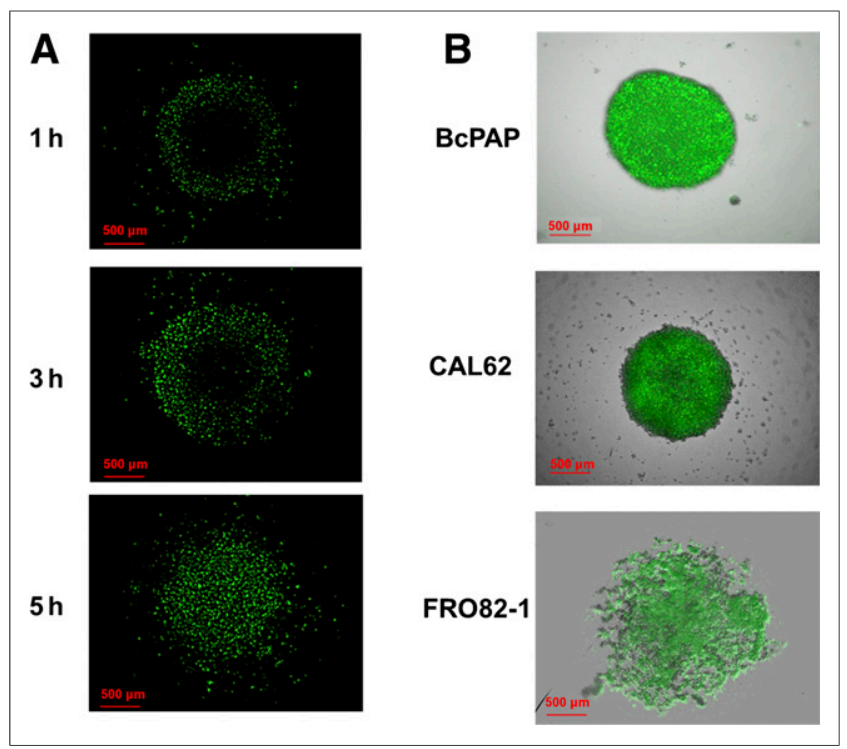

FIGURE 3. Characterization of gal-3-specific $F\left(a b^{\prime}\right) 2$ binding to spheroids. (A) Time-dependent penetration of AlexaFluor $488-\mathrm{aGal} 3-\mathrm{F}\left(\mathrm{ab}^{\prime}\right)_{2}$ into BcPAP tumor spheroids during incubation with $10 \mu \mathrm{g} / \mathrm{mL}$ concentration of AlexaFluor 488 conjugate (representative image). (B) Full focus confocal and fluorescent overlay of tumor spheroids.

than the gal-3 band but 3-fold stronger for FRO82-1 and BcPAP cells than for CAL62 cells (Supplemental Fig. 1A).

A high Gal-3 messenger RNA expression in 2D cell cultures was detectable compared with normal human thyrocytes, being higher in

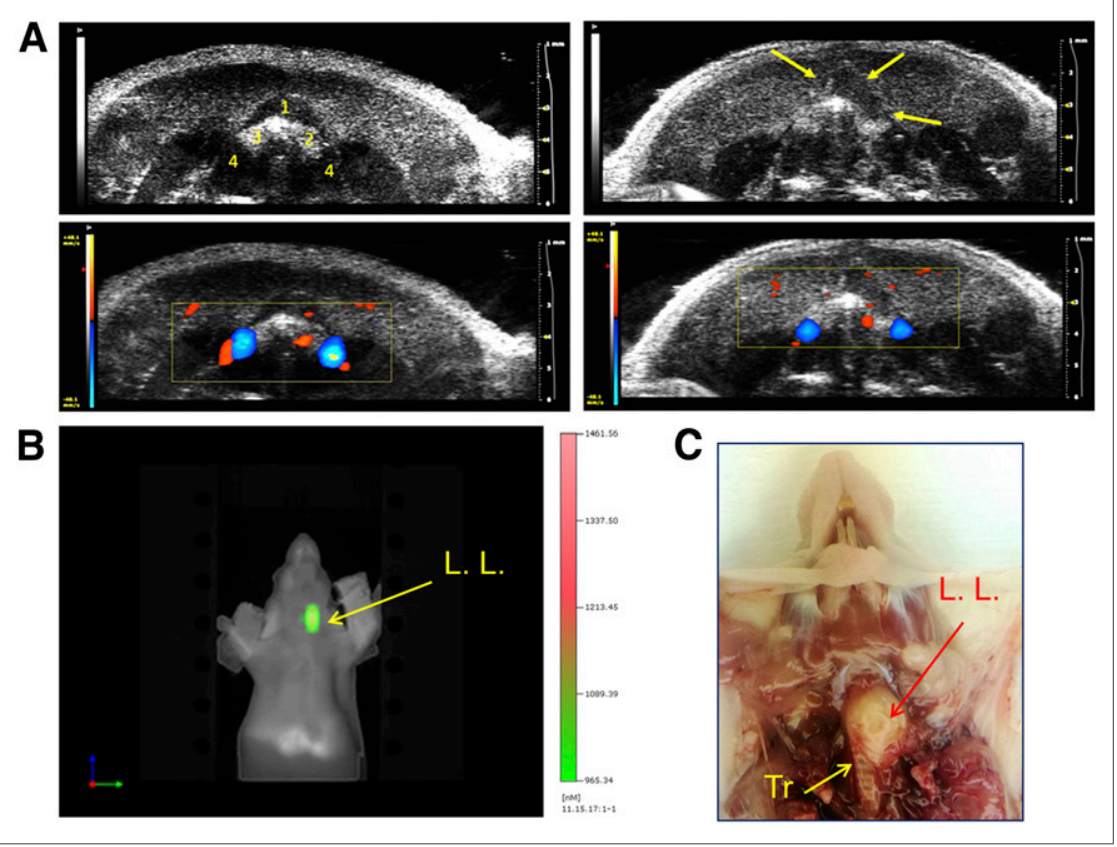

FIGURE 4. Orthotopic tumor growth monitoring. (A) Representative ultrasound transversal image of mouse neck before thyroid cancer cell transplantation (top left): strap muscle (1), left thyroid lobe (2), right thyroid lobe (3), carotid arteries (4); ultrasound image showing orthotopic tumor (arrows) expanding from left lobe (top right); and Doppler mode enhancement of activity of carotid arteries and correct localization of thyroid (bottom). (B) FMT imaging of same mouse performed $48 \mathrm{~h}$ after injection of Cy5.5-labeled aGal3-F(ab') ${ }_{2}$ and visualizing gal-3-positive mass in neck. (C) Anatomic analysis of thyroid orthotopic tumor at necropsy. $\operatorname{Tr}=$ trachea; L. L. $=$ left lobe. tumor spheroids. Conversely, human NIS messenger RNA was undetectable in 2D and 3D cell cultures compared with the expression in normal human thyrocytes (Fig. 1B; Supplemental Figs. 1B and 1C).

The presence of a thyroid transcription factor 1 double band at $40 \mathrm{kDa}$ confirmed the phenotype of the tumor cell lines (Fig. 1A).

\section{lodine Uptake in 2D Cell Cultures and Tumor Spheroids}

${ }^{125}$ I uptake in monolayer cultures and tumor spheroids was strongly dependent on the differential human NIS expression evidenced via Western blot and PCR analysis. ${ }^{125}$ I internalization in CAL62 was very low when compared with the higher accumulation in FRO82-1 and BcPAP cells (Fig. 2A, left). A specular ${ }^{125} \mathrm{I}$ accumulation was measured on tumor spheroids (Fig. 2A, right).

\section{Characterization of Gal-3 Tracers and Cell Binding}

Radiolabeling of the antigal-3 $\mathrm{F}\left(\mathrm{ab}^{\prime}\right)_{2}$ with ${ }^{89} \mathrm{Zr}$ provided a probe $\left({ }^{89} \mathrm{Zr}-\mathrm{DFO}-\mathrm{aGal} 3-\mathrm{F}\left(\mathrm{ab}^{\prime}\right)_{2}\right)$ with $29.6 \pm 2.0 \mathrm{GBq} / \mu \mathrm{mol}$ specific activity, more than $98 \%$ radiochemical purity measured via radio-high-performance liquid chromatography (Supplemental Fig. 2A), retained integrity, and high stability in human serum (Supplemental Figs. 2B and 2C).

A dissociation constant of $3.9 \pm 0.2 \mathrm{nM}$ and an immunoreactivity of $75.2 \%$ (Figs. $2 \mathrm{~B}$ and $2 \mathrm{C}$ ) were measured. In internalization tests, $30 \%$ of the added ${ }^{89} \mathrm{Zr}-\mathrm{DFO}-\mathrm{aGal} 3-\mathrm{F}\left(\mathrm{ab}^{\prime}\right)_{2}$ was internalized after 5 min of incubation, reaching 62\% (Supplemental Fig. 2D) in the remaining $120 \mathrm{~min}$. A size-dependent binding of ${ }^{89} \mathrm{Zr}$-DFO-aGal3$\mathrm{F}\left(\mathrm{ab}^{\prime}\right)_{2}$ to tumor spheroids was measured (Supplemental Fig. 3A).

A time-dependent penetration of the AlexaFluor 488-conjugated probe $\left(5.0 \pm 0.1\right.$ dye particles per molecule of $\left.\mathrm{F}\left(\mathrm{ab}^{\prime}\right)_{2}\right)$ and a strong fluorescent signal within $5 \mathrm{~h}$ of incubation were evidenced (Fig. $3 \mathrm{~A}$ ), in particular for more compact tumor spheroids (BcPAP and CAL62) compared with cell aggregates (FRO82-1) (Fig. 3B).

The spheroids showed viability and metabolic activity, indirectly measured by the reduction of the water-soluble tetrazolium salt 1 (Supplemental Fig. 3B), a reaction catalyzed by cell-surface nicotinamideadenine-dinucleotide-plus-hydrogen (NAPDH) oxidases (16,17). All cell lines showed substantial NAPDH oxidase activity, indicating an active metabolism (18). However, compared with BcPAP and CAL62, the metabolic activity per cell for FRO82-1 spheroidlike structures was about 3-fold higher.

\section{Orthotopic Thyroid Tumor Growth Monitoring}

The mice tolerated the orthotopic transplantation of cancer cells well, without any complication related to surgery or anesthesia. BcPAP, FRO82-1, and CAL62 cell lines exhibited high tumorigenicity $(>90 \%)$ after intrathyroid injection, and there was a difference in the rate of tumor volume growth among FRO82-1, CAL62, and BcPAP, which reached a diameter of $0.3-0.5 \mathrm{~cm}$ in 3,5 , and $6 \mathrm{wk}$, respectively, after cell transplantation.

The growth of tumors was visualized by ultrasound imaging as a small dark area within the left thyroid (Fig. 4A). The neoplastic nature of this structure was confirmed by 


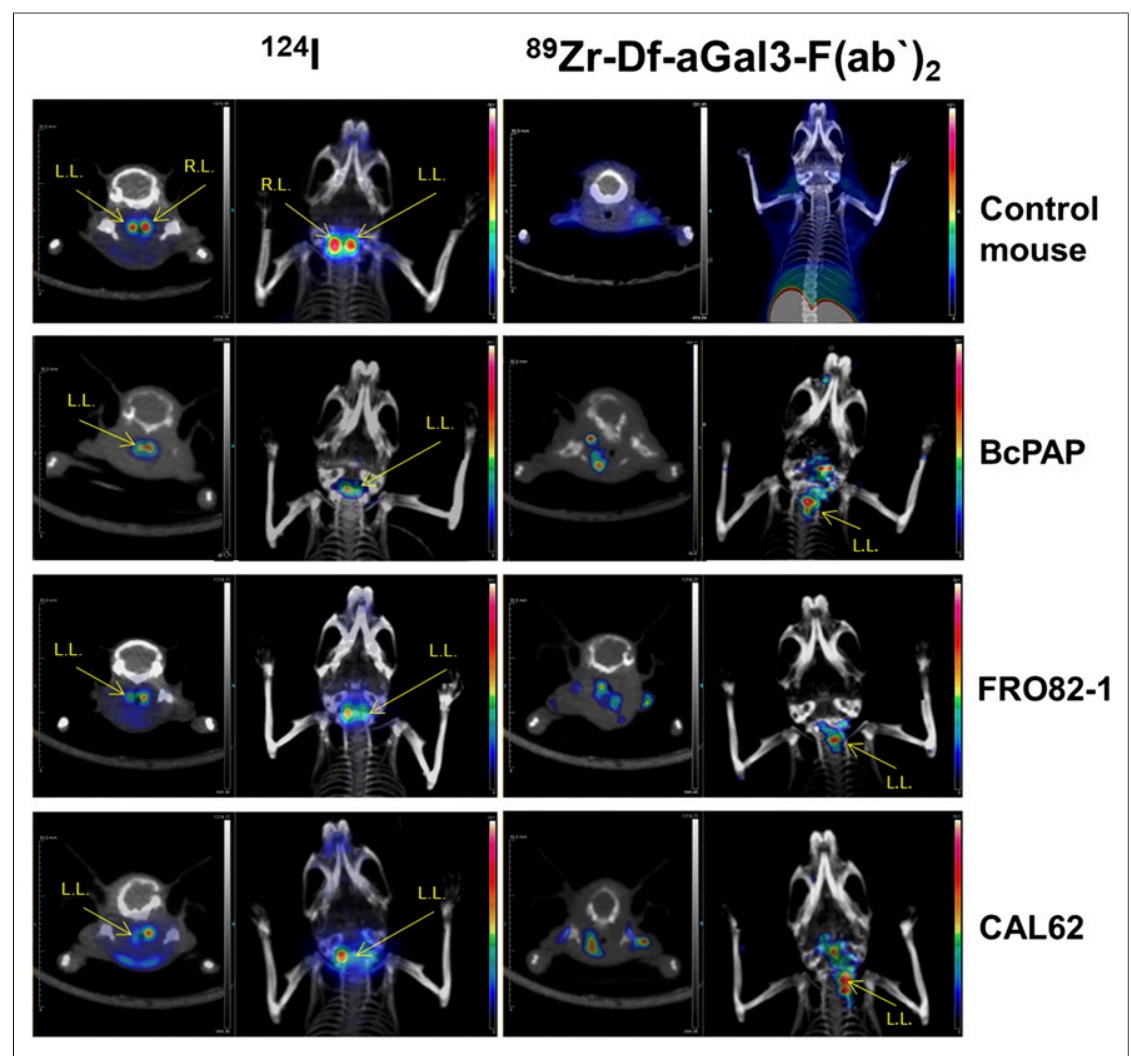

FIGURE 5. Head-to-head comparison of ${ }^{124}$ vs. ${ }^{89} \mathrm{Zr}$-DFO-aGal3-F(ab' ${ }_{2}$ detection of thyroid orthotopic tumors. Representative small-animal PET images were acquired $1 \mathrm{~h}$ after injection of ${ }^{124} \mathrm{I}$ and $48 \mathrm{~h}$ after injection of ${ }^{89} \mathrm{Zr}$-DFO-aGal3-F( $\left(\mathrm{ab}^{\prime}\right)_{2}$. Each row presents axial PET/CT fusion projection and $3 \mathrm{D}$ projection for both tracers. L. L. = left lobe; R. L. = right lobe.

Head-to-Head PET/CT Comparison of Gal-3 Immunotargeting Versus ${ }^{124}$

In control mice injected with ${ }^{124} \mathrm{I}-\mathrm{NaI}$, radioiodine accumulation in both thyroid lobes was visible after $1 \mathrm{~h}$ (Fig. 5, left). Control mice injected with ${ }^{89} \mathrm{Zr}$-DFO-aGal3$\mathrm{F}\left(\mathrm{ab}^{\prime}\right)_{2}$ did not show any accumulation in normal thyroid (Fig. 5, right; Supplemental Fig. 4A). Mice bearing tumors showed strong accumulation of ${ }^{124} \mathrm{I}$ in the right thyroid, with a weaker and a decreasing signal in the left thyroid, for BcPAP > FRO82-1 > CAL62, respectively (Fig. 5, left). Conversely, a strong signal associated only with the left thyroid was visible in all mouse models injected with ${ }^{89} \mathrm{Zr}$-DFO-aGal3-F $\left(\mathrm{ab}^{\prime}\right)_{2}$ (Fig. 5, right; Supplemental Fig. 4B).

In some animals, the enlargement of the thyroid lobe during tumor cell injection induced extravasation of medium-containing cells, which infiltrated the neck fatty tissues. Those cells were not visualized via ${ }^{124} \mathrm{I}$ PET/CT but were highlighted using ${ }^{89} \mathrm{Zr}-\mathrm{DFO}-\mathrm{aGal} 3-\mathrm{F}\left(\mathrm{ab}^{\prime}\right)_{2}$.

\section{Tracer Accumulation Analysis}

A high ${ }^{124}$ I retention was measured in the right thyroid $(42.4 \pm 5.6 \% \mathrm{ID} / \mathrm{g}) \mathrm{com}$ pared with the left thyroid $(6.6 \pm 2.0,2.7$ \pm 1.1 , and $1.7 \pm 0.1 \% \mathrm{ID} / \mathrm{g}$ for BcPAP, FRO82-1, and CAL62, respectively), confirming a reduced tumor NIS expression (Fig. 6A). High accumulation of ${ }^{89} \mathrm{Zr}-$ DFO-aGal3-F $\left(\mathrm{ab}^{\prime}\right)_{2}$ was measured in the

FMT imaging in the neck region using Cy5.5-conjugated $\mathrm{F}\left(\mathrm{ab}^{\prime}\right)_{2}$ to gal-3 (3.6 \pm 0.1 dye particles per molecule of $\left.\mathrm{F}\left(\mathrm{ab}^{\prime}\right)_{2}\right)$ (Fig. 4B). The position of the tumor was confirmed at anatomic analysis (Fig. 4C). left thyroid $(7.2 \pm 0.9,3.9 \pm 0.5$, and $4.2 \pm 1.3 \% \mathrm{ID} / \mathrm{g}$ for FRO82-1, BcPAP, and CAL62, respectively; Fig. 6B), compared with right thyroid $(1.3 \pm 0.3 \% \mathrm{ID} / \mathrm{g})$.

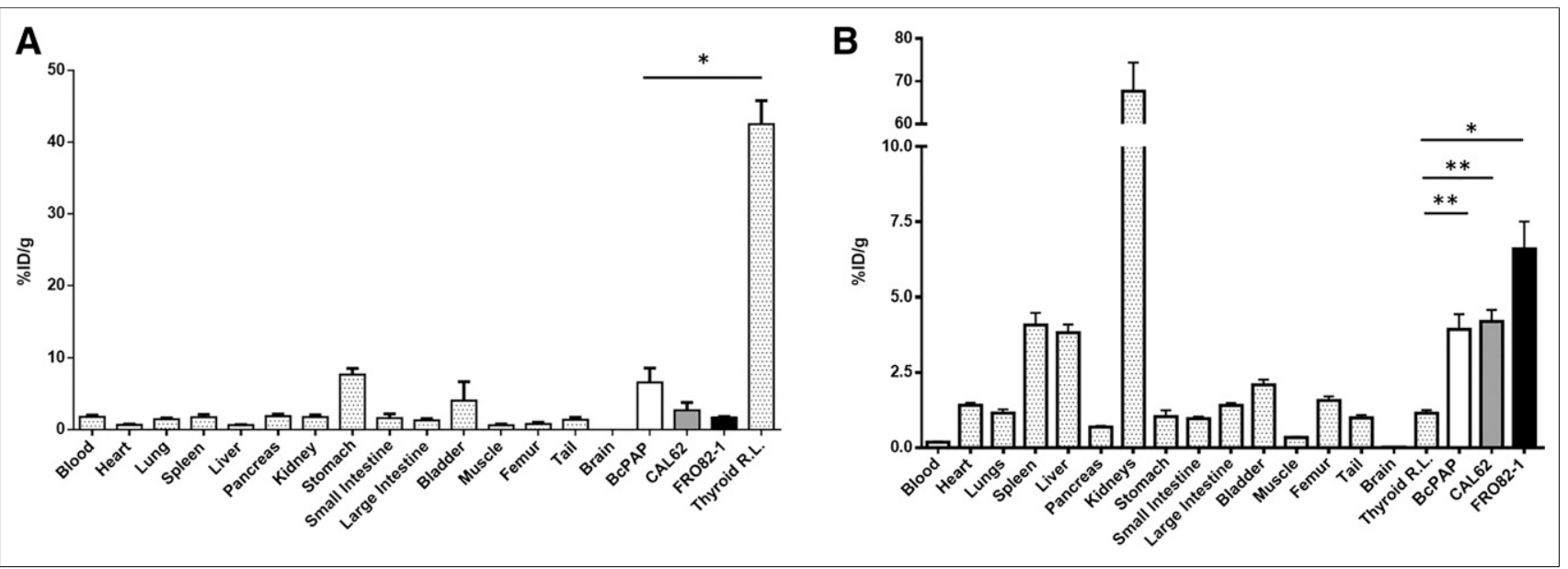

FIGURE 6. Biodistribution analysis of ${ }^{124} \mid$ vs. ${ }^{89} \mathrm{Zr}$-DFO-aGal3-F(ab') . Three groups of mice (3 per tumor type) were injected with ${ }^{124} \mathrm{I}$ and 2 groups of mice (5 per tumor type) were injected with ${ }^{89} \mathrm{Zr}$-DFO-aGal3-F(ab') ${ }_{2}$. (A) Low NIS expression in orthotopic tumors yielded low ${ }^{124}$ accumulation in left lobe compared with right lobe. Differences were statistically significant $\left({ }^{*} P<0.01\right)$. (B) Strong ${ }^{89} \mathrm{Zr}-\mathrm{DFO}-\mathrm{aGal} 3-\mathrm{F}\left(\mathrm{ab}{ }^{\prime}\right)_{2}$ retention was measured in orthotopic tumors, with background accumulation in right lobes. Differences in ${ }^{89} \mathrm{Zr}-\mathrm{DFO}-\mathrm{aGal} 3-\mathrm{F}\left(\mathrm{ab} \mathrm{b}_{2}\right)_{2}$ accumulation were statistically significant ( ${ }^{\star} P=<0.01$ for FRO82-1; ${ }^{\star *} P<0.05$ for BcPAP and CAL62). R. L. = right lobe. 


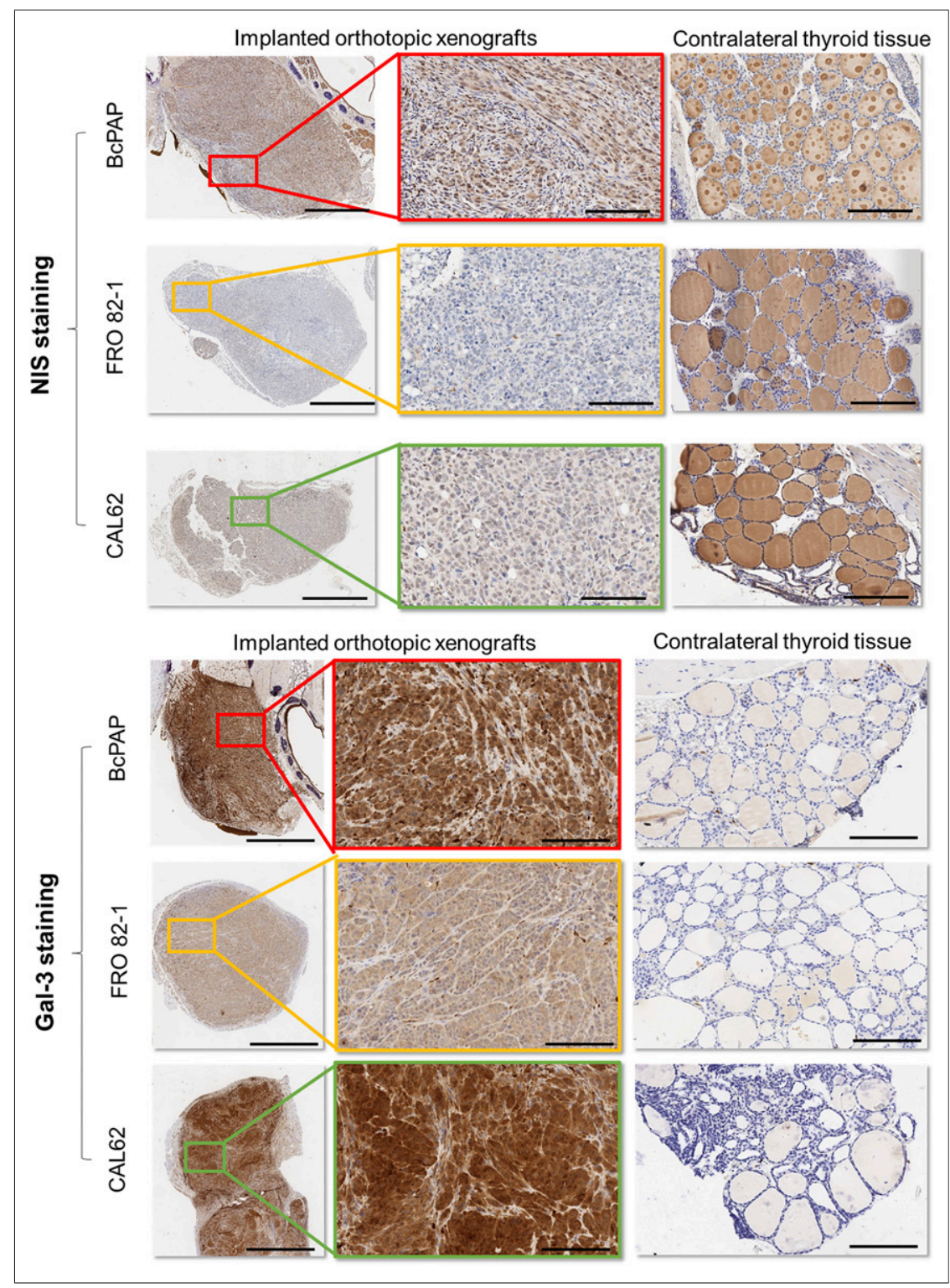

FIGURE 7. Immunohistochemical analysis for NIS and gal-3 expression. Tissue sections derived from normal and tumor-infiltrated thyroid lobes were stained for NIS and gal-3 expression using rabbit polyclonal antibody anti-NIS (GTX37599, $0.5 \mathrm{mg} / \mathrm{mL}$; Genetex) and horseradish peroxidase-conjugated rat mAb to gal-3 (Mabtech) $(10 \mu \mathrm{g} / \mathrm{mL}$ ). (Top) Insets show weak membrane staining on tumor cells, and right column shows normal lobe with thyrocytes positive for NIS staining. (Bottom) On Gal-3 staining, insets show cytoplasmic staining, and right column shows normal lobe without any visible signal for gal-3. Left $=\times 4$ with $2.0-\mathrm{mm}$ bar; middle $=\times 20$ with $300-\mu \mathrm{m}$ bar; right $=\times 10$ with 1 -mm bar.

A low left-thyroid-to-right-thyroid ${ }^{124} \mathrm{I}$ accumulation ratio $(0.16 \pm 0.02,0.06 \pm 0.01$, and $0.04 \pm 0.01$ for BcPAP, FRO82-1, and CAL62, respectively) was measured (Supplemental Fig. 5A), compared with the ${ }^{89} \mathrm{Zr}-\mathrm{DFO}-\mathrm{aGal} 3-\mathrm{F}\left(\mathrm{ab}^{\prime}\right)_{2}$ accumulation ratio (4.0 $\pm 0.7,5.5 \pm 1.5$, and $3.5 \pm 0.9$ for BcPAP, FRO82-1, and CAL62, respectively; Supplemental Fig. 5B; Supplemental Tables 2 and 3), which yielded high-contrast images. ${ }^{89} \mathrm{Zr}-\mathrm{DFO}-\mathrm{aGal} 3-\mathrm{F}\left(\mathrm{ab}^{\prime}\right)_{2}$ retention in liver and spleen can be explained by ${ }^{89} \mathrm{Zr}$ residualization after catabolism of the conjugate (19) but in kidneys is due to tracer excretion $(77.0 \pm 15.0 \% \mathrm{ID} / \mathrm{g})$.

The exposure for the kidneys resulted in an organ dose of 0.01 $\mathrm{mSv} / \mathrm{MBq}$, versus an estimated effective total-body dose of 0.061
$\mathrm{mSv} / \mathrm{MBq}$. A good correlation $\left(R^{2}=0.69\right)$ between image-derived uptake calculation and ex vivo accumulation analysis was found (Supplemental Fig. 5C).

\section{Immunohistochemical Analysis of Tumor Xenografts and Normal Mouse Thyroid}

Immunostaining for NIS expression revealed a stronger signal in BcPAP tumors and a weaker signal for FRO82-1 and a CAL62 (Fig. 7, top inset). Conversely, a high NIS expression was visualized for the contralateral right thyroid (as expected), with thyrocytes positive for NIS around the thyroidal follicles (Fig. 7, top right).

Gal-3 expression was detected in the cytoplasm of the thyroid cancer cells infiltrating the left thyroid (Fig. 7, bottom inset), with stronger staining for BcPAP and CAL62 tumors than for FRO82-1. Gal-3 was undetectable in normal residual parenchyma of the left thyroid and in the tumorfree right thyroid (Fig. 7, bottom right).

\section{DISCUSSION}

Thyroid nodules are common in adults, especially in areas with iodine deficiency. Depending on the ultrasound technology, the prevalence in healthy populations ranges between $33 \%$ and $68 \%$ (20). Currently, it is often not possible to exclude a malignant nodule by imaging and fine-needle aspiration biopsy, especially in patients with multinodular goiter, because an imaging agent with high and specific uptake in malignant lesions is lacking. This uncertainty results in a large number of thyroidectomies for benign thyroid diseases, with a ratio of 1:15 malignant versus benign nodules in Germany, 1:7 in Italy, and 1:5 in Great Britain (21). A noninvasive imaging test that specifically accumulates in malignant thyroid nodules could substantially reduce the morbidity from thyroid surgeries for benign nodules and reduce health-care costs (22).

The specific expression of gal-3 by thyroid cancer makes it an excellent target for the development of such an imaging test $(5,6,23,24)$. In fact, gal-3 staining of thyroid fine-needle aspiration biopsy samples has already been shown to be able significantly reduce the number of surgeries for benign thyroid nodules. However, fine-needle aspiration is limited by sampling errors (especially in multinodular goiters), which can be overcome by molecular imaging targeting gal-3. Herein, we therefore performed the preclinical evaluation of gal-3 immunotargeting with ${ }^{89} \mathrm{Zr}-\mathrm{DFO}-\mathrm{aGal} 3-\mathrm{F}\left(\mathrm{ab}^{\prime}\right)_{2}$ for detection of malignant thyroid nodules. $\mathrm{F}(\mathrm{ab})_{2}$ fragments were used because of their faster blood clearance and lower liver uptake than monoclonal antibodies $(6,19,25)$.

We started our evaluation with tumor spheroids, in vitro cellular models that better mimic the physiologic tissue characteristics (e.g., 
cell-cell interaction), which help in predicting the in vivo results, especially for cell adhesion molecules such as gal-3 (12,26). According to Western blot and quantitative PCR analysis, the 2D and 3D cell cultures showed low to absent human NIS expression and high gal-3 expression, thus explaining the differential accumulation of ${ }^{125} \mathrm{I}$ on monolayer and spheroid cultures, and the specific binding of ${ }^{89} \mathrm{Zr}$-DFO-aGal3-F $\left(\mathrm{ab}^{\prime}\right)_{2}$ determined by an active internalization process. The combination of ultrasound and FMT imaging to monitor the orthotopic tumor growth suggests the possibility to use gal3-specific probes conjugated to near-infrared dye $(700-900 \mathrm{~nm})$ for performing image-guided surgery of thyroid nodules (27-29).

The head-to-head comparison of ${ }^{124}$ I PET/CT versus gal-3 targeting demonstrated the specificity of our methodology for thyroid cancer imaging. High uptake of ${ }^{89} \mathrm{Zr}-\mathrm{DFO}-\mathrm{aGal} 3-\mathrm{F}\left(\mathrm{ab}^{\prime}\right)_{2}$ was observed for the orthotopic thyroid tumors that showed only minimal or no radioiodine uptake. Conversely, the normal thyroid demonstrated high radioiodine uptake but no specific uptake of ${ }^{89} \mathrm{Zr}$-DFO$\mathrm{aGal3}-\mathrm{F}\left(\mathrm{ab}^{\prime}\right)_{2}$. These imaging findings were confirmed by ex vivo biodistribution studies for ${ }^{89} \mathrm{Zr}-\mathrm{DFO}-\mathrm{aGal} 3-\mathrm{F}\left(\mathrm{ab}^{\prime}\right)_{2}$. Lack of ${ }^{89} \mathrm{Zr}$ DFO-aGal3-F $\left(\mathrm{ab}^{\prime}\right)_{2}$ uptake by the normal thyroid tissue is not due to species differences since the $\mathrm{F}\left(\mathrm{ab}^{\prime}\right)_{2}$ recognizes an amino-terminal common epitope of human and mouse gal-3 $(23,24)$.

The high uptake of ${ }^{89} \mathrm{Zr}-\mathrm{DFO}-\mathrm{aGal} 3-\mathrm{F}\left(\mathrm{ab}^{\prime}\right)_{2}$ in thyroid tumors without significant radioiodine uptake also indicates that gal-3 is a promising target for staging and potentially for radionuclide therapy of radioiodine-negative or refractory thyroid cancer. The high kidney uptake of ${ }^{89} \mathrm{Zr}$-DFO-aGal3-F( $\left(\mathrm{ab}^{\prime}\right)_{2}$ is a limitation for therapeutic applications. However, it may be reduced by coadministration of cationic amino acid solutions or basic polypeptides (poly-lysine) (30). In addition, modifications of antibody fragments by PEGylation have been shown to drastically reduce renal uptake (31).

\section{CONCLUSION}

Using an antigal-3 $\mathrm{F}\left(\mathrm{ab}^{\prime}\right)_{2}$, we demonstrated that molecular imaging of gal-3 expression is a new tool for in vivo detection of thyroid cancer. Our results are promising for noninvasive identification of malignant thyroid nodules with nuclear and optical imaging, staging of thyroid cancer, and targeted radionuclide therapy of metastatic, radioiodine-negative thyroid cancer.

\section{DISCLOSURE}

This study was funded by Deutsche Forschungsgemeinschaft (grant DA 1552/2-1), SFB824 (Z2), and SFB824 (C10). No other potential conflict of interest relevant to this article was reported.

\section{ACKNOWLEDGMENTS}

We thank Michael Kuhlmann from the EIMI of WestfälischeWilhelms-Universität in Münster for teaching intrathyroidal tumor cell implantation; Markus Mittelhäuser for injecting tracer in the animals; Marion Mielke for supporting us with tissue immunostaining; Joseph Hintermair for helping with spheroid establishment and water-soluble tetrazolium salt 1 analysis; and Stephanie Rämisch for assisting with RNA isolation.

\section{REFERENCES}

1. Morris LG, Tuttle RM, Davies L. Changing trends in the incidence of thyroid cancer in the United States. JAMA Otolaryngol Head Neck Surg. 2016;142:709-711.

2. Siegel RL, Miller KD, Jemal A. Cancer statistics, 2018. CA Cancer J Clin. 2018;68:7-30.

3. Nikiforov YE, Seethala RR, Tallini G, et al. Nomenclature revision for encapsulated follicular variant of papillary thyroid carcinoma: a paradigm shift to reduce overtreatment of indolent tumors. JAMA Oncol. 2016;2:1023-1029.
4. Kogai T, Brent GA. The sodium iodide symporter (NIS): regulation and approaches to targeting for cancer therapeutics. Pharmacol Ther. 2012;135:355-370.

5. Bartolazzi A, D'Alessandria C, Parisella MG, et al. Thyroid cancer imaging in vivo by targeting the anti-apoptotic molecule galectin-3. PLoS One. 2008;3:e3768.

6. D'Alessandria C, Braesch-Andersen S, Bejo K, et al. Noninvasive in vivo imaging and biologic characterization of thyroid tumors by immunoPET targeting of galectin-3. Cancer Res. 2016;76:3583-3592.

7. Livak KJ, Schmittgen TD. Analysis of relative gene expression data using realtime quantitative PCR and the $2^{-\Delta \Delta C_{\mathrm{T}}}$ method. Methods. 2001;25:402-408.

8. Rousseaux J, Rousseaux-Prevost R, Bazin H. Optimal conditions for the preparation of $\mathrm{Fab}$ and $\mathrm{F}\left(\mathrm{ab}^{\prime}\right) 2$ fragments from monoclonal IgG of different rat $\mathrm{IgG}$ subclasses. J Immunol Methods. 1983;64:141-146.

9. Inohara H, Raz A. Functional evidence that cell surface galectin-3 mediates homotypic cell adhesion. Cancer Res. 1995;55:3267-3271.

10. Yusufi N, Mall S, Bianchi HO, et al. In-depth characterization of a TCR-specific tracer for sensitive detection of tumor-directed transgenic $\mathrm{T}$ cells by immunoPET. Theranostics. 2017;7:2402-2416.

11. Sham JG, Kievit FM, Grierson JR, et al. Glypican-3-targeted ${ }^{89} \mathrm{Zr}$ PET imaging of hepatocellular carcinoma. J Nucl Med. 2014;55:799-804.

12. Singh TD, Jeong SY, Lee SW, et al. Inverse agonist of estrogen-related receptor gamma enhances sodium iodide symporter function through mitogen-activated protein kinase signaling in anaplastic thyroid cancer cells. J Nucl Med. 2015;56:1690-1696.

13. Lindmo T, Cuttitta F, Fedorko J, Bunn PA Jr. Determination of the immunoreactive fraction of radiolabeled monoclonal antibodies by linear extrapolation to binding at infinite antigen excess. J Immunol Methods. 1984;72:77-89.

14. Büther K, Compeer MG, De Mey JG, et al. Assessment of endothelin-A receptor expression in subcutaneous and orthotopic thyroid carcinoma xenografts in vivo employing optical imaging methods. Endocrinology. 2012;153:2907-2918.

15. Stabin MG, Sparks RB, Crowe E. OLINDA/EXM: the second-generation personal computer software for internal dose assessment in nuclear medicine. $\mathrm{J} \mathrm{Nucl}$ Med. 2005;46:1023-1027.

16. Otto AM, Paddenberg R, Schubert S, Mannherz HG. Cell-cycle arrest, micronucleus formation, and cell death in growth inhibition of MCF-7 breast cancer cells by tamoxifen and cisplatin. J Cancer Res Clin Oncol. 1996;122:603-612.

17. Berridge MV, Herst PM, Tan AS. Tetrazolium dyes as tools in cell biology: new insights into their cellular reduction. Biotechnol Annu Rev. 2005;11:127-152.

18. Otto AM, Hintermair J, Janzon C. NADH-linked metabolic plasticity of MCF-7 breast cancer cells surviving in a nutrient-deprived microenvironment. $J$ Cell Biochem. 2015;116:822-835.

19. Anderson CJ, Connett JM, Schwarz SW, et al. Copper-64-labeled antibodies for PET imaging. J Nucl Med. 1992;33:1685-1691.

20. Guth S, Theune U, Aberle J, Galach A, Bamberger CM. Very high prevalence of thyroid nodules detected by high frequency (13 MHz) ultrasound examination. Eur J Clin Invest. 2009;39:699-706.

21. Rosato L, Avenia N, Bernante P, et al. Complications of thyroid surgery: analysis of a multicentric study on 14,934 patients operated on in Italy over 5 years. World J Surg. 2004;28:271-276.

22. Scott-Combes D, Kinsman R. The British Association of Endocrine Surgeons: Second National Audit Report 2007. Oxfordshire, United Kingdom: Dendrite Clinical Systems Ltd; 2007.

23. Bartolazzi A, Gasbarri A, Papotti M, et al. Application of an immunodiagnostic method for improving preoperative diagnosis of nodular thyroid lesions. Lancet. 2001;357:1644-1650.

24. Bartolazzi A, Orlandi F, Saggiorato E, et al. Galectin-3-expression analysis in the surgical selection of follicular thyroid nodules with indeterminate fine-needle aspiration cytology: a prospective multicenter study. Lancet Oncol. 2008;9:543-549.

25. Sham JG, Kievit FM, Grierson JR, et al. Glypican-3-targeting $F\left(a^{\prime}\right) 2$ for ${ }^{89} \mathrm{Zr}$ PET of hepatocellular carcinoma. J Nucl Med. 2014;55:2032-2037.

26. Fortunati N, Catalano MG, Arena K, Brignardello E, Piovesan A, Boccuzzi G. Valproic acid induces the expression of the $\mathrm{Na}+/ \mathrm{I}-$ symporter and iodine uptake in poorly differentiated thyroid cancer cells. J Clin Endocrinol Metab. 2004;89:1006-1009.

27. Li Y, Li Z, Wang X, et al. In vivo cancer targeting and imaging-guided surgery with near infrared-emitting quantum dot bioconjugates. Theranostics. 2012;2:769-776.

28. Kahramangil B, Berber E. The use of near-infrared fluorescence imaging in endocrine surgical procedures. J Surg Oncol. 2017;115:848-855.

29. Vahrmeijer AL, Hutteman M, van der Vorst JR, van de Velde CJ, Frangioni JV. Image-guided cancer surgery using near-infrared fluorescence. Nat Rev Clin Oncol. 2013;10:507-518.

30. Behr TM, Goldenberg DM, Becker W. Reducing the renal uptake of radiolabeled antibody fragments and peptides for diagnosis and therapy: present status, future prospects and limitations. Eur J Nucl Med. 1998;:25:201-212.

31. Rashidian M, Ingram JR, Dougan M, et al. Predicting the response to CTLA-4 blockade by longitudinal noninvasive monitoring of CD8 T cells. J Exp Med. 2017; 214:2243-2255. 